\title{
动作发展神经科学: 未来路径与布局
}

\author{
武志俊 ${ }^{1,2}$, 王争艳 ${ }^{1,2 *}$, 王强 ${ }^{1,2}$
}

1. 首都师范大学心理学院, 北京市“学习与认知”重点实验室, 北京 100048 ;

2. 首都师范大学心理学院, 首师大儿童发展研究中心, 北京 100048

*联系人, E-mail: wangzhengyan@cnu.edu.cn

收稿日期: 2020-12-02; 接受日期: 2021-02-24; 网络版发表日期: 2021-04-27

中国科学技术协会学科发展项目(批准号: 2018XKFZ03)和国家自然科学基金(批准号: 31971006)资助

摘要儿童早期动作的发展可视为个体早期的外显智力, 动作的毕生发展与个体认知发展及个性和社会性发展 密切相关. 认知神经学科和脑科学技术的革命性发展为研究动作领域的毕生发展的神经机制提供了基础和契机. 从毕生发展的角度看, 动作的产生以及动作技能的获得是婴儿阶段发育的里程碑; 到了儿童青少年期, 动作技能 的有效性、精确性和稳定性均得到提高; 成年早期到晚期, 运动能力经历了巅峰到明显衰退的转变. 目前关于动 作的神经机制的研究主要探究了毕生发展过程中动作的产生、发展、衰退背后的大脑结构变化及大脑网络连接 的改变. 本文从认知科学和认知神经科学角度回顾了个体动作在婴幼儿期、儿童青少年期及成年期等阶段的发 展特点及其背后的神经机制, 提出了未来该领域认知神经科学方向面临的挑战及亟待解决的问题, 并对国内该领 域未来的研究和发展进行了分析和预测.

关键词 动作, 毕生发展, 认知心理, 认知神经

动作的发展被视为贞儿发展的重要里程碑. 动作 行为是个体自适应功能中最早可直接观察到的元素之 - ${ }^{[1]}$. 儿童心理学家皮亚杰将动作视为个体心理的起 源, 认为个体心理发展是主体通过动作对环境的适 应 $^{[2]}$. 儿童早期的动作发展可分为粗大动作发展和精 细动作发展. 粗大动作和精细动作的出现标志着贞儿 自主能力的增强, 这使得贞儿能以越来越复杂的方式 对环境采取行动并与之互动, 并为环境探索、感知学 习和社交互动提供了更多机会 ${ }^{[3,4]}$. 儿童早期动作的发 展亦是认知发展的外在表现，可视为个体早期的外显 智力 ${ }^{[5]}$, 并且早期动作的良好发展也将促使儿童的认
知发展不断高级化、复杂化 ${ }^{[6]}$. 大脑的发育是动作发 展的基础，同时动作的发展也会促进大脑的进一步发 展. 在反复的动作练习过程中, 大脑相应区域不断被 激活, 从而使大脑结构产生积极性变化, 为个体心理 发展奠定良好的神经基础 ${ }^{[7-9]}$. 在毕生发展过程中, 动 作的产生、发展和老化衰退与大脑结构和功能的发展 密切相关. 认知神经科学的发展, 特别是无损伤脑影像 技术的出现和发展, 为发展心理学与脑科学的结合提 供了技术上的可行性，拓宽了人们对大脑结构和大脑 功能的认识. 基于此, 从认知神经科学的角度, 探明动 作的毕生发展规律和特点, 探究动作发生的神经机制,

引用格式: 武志俊, 王争艳, 王强. 动作发展神经科学: 未来路径与布局. 中国科学: 生命科学, 2021, 51: 619-633

Wu Z J, Wang Z Y, Wang Q. The neuroscience of motor development: the future path and layout (in Chinese). Sci Sin Vitae, 2021, 51: 619-633, doi: $10.1360 /$ SSV-2020-0242 
将有助于与动作相关的各个学科之间的整合, 也有助 于更加全面深入地理解动作发展的心理意义.

本文将结合国内外研究, 梳理动作在毕生发展各 个阶段的行为发展特点, 并从认知神经发展的角度梳 理动作毕生发展的神经机制的相关研究，最后对我国 当前该领域研究面临的挑战进行分析，并提出该领域 未来的发展规划.

\section{1 动作的毕生发展}

本文根据身心发展趋势，将毕生发展的阶段划为 以下 3 个主要的阶段：婴幼儿期( $0 \sim 7$ 岁 $)$ 、儿童青少年 期(7 18岁)和成年期(18岁之后 $)^{[2]}$. 其中, 成年期又划 分为 3 个子阶段：成年早期 $(18 \sim 35$ 岁)、成年中期 (35 60岁)和成年晚期(60岁以后) ${ }^{[10,11]}$.

\section{1 婴幼儿期的动作发展}

婴儿出生后第一年最为引人注目的一个变化, 就 是婴儿在控制自身运动和动作技能方面的巨大进步. 贞幼儿期的早期运动发育对儿童日后的身体、认知、 情绪和社会结果都有显著的影响 ${ }^{[3,12 \sim 14]}$.

贞儿动作技能的发展包括粗大动作技能和精细动 作技能两方面. 粗大动作技能是指在进行大幅度身体 运动时表现出来的能力, 如抬头、爬行、站立、走路 和跳跃等. 精细动作技能指通过手臂、手和手指对细 小动作控制时表现出的能力，如自主够物、抓握、书 写、绘画以及生活技能(穿衣、进食等)等.

婴幼儿动作技能发展按照一定的原则有序进 行 $^{[5,15]}$. 头尾原则指动作技能的发展按从头到脚、由 上至下的顺序进行. 近远原则指动作技能的发展按由 中央到外围、由近及远的顺序进行. 大小原则指动作 技能的发展是从涉及大肌肉的粗大动作逐渐过渡到涉 及小肌肉的精细动作.

在粗大动作技能发展方面，新生儿的动作技能非 常有限，最重要的能力就是拥有一整套的反射．新生 儿一出生或出生后不久会表现出多种反射。6个月时, 婴儿可以让自己向特定的方向移动, 到6 8个月, 婴儿 基本掌握爬行的技能，在 9 个月时，大多数贞儿能借助 桌椅走路, 1岁时, 大约一半的婴儿能走得很好. 1 2 岁 学步跑动时步频大、步幅小、呈跳跃式，不能很自如 地转弯或停止. 随着儿童的成熟, 他们的动作技能提
高得非常迅速，跑、爬、跳跃和投掷都进步很大. 3 岁 时, 他们已经可以沿直线走或跑, 能双脚离地在地板上 跳. 3 6岁是幼儿动作发展的关键时期, 个体在这一阶 段形成多种基本动作技能 ${ }^{[16]}$. 3.5 4岁的儿童大致能完 成单脚跳的动作, 然而连续跳跃的次数少, 且水平移动 的距离很短. 4.5 5岁儿童单脚跳时, 支撑腿的力量有 所增加, 连续跳跃的次数增多. 5 岁时, 儿童的动作变得 相当熟练, 在跑动时, 他们可以像成人一样摆动胳膊, 其平衡能力已经提高到相当高的水平. 幼儿期粗大动 作技能的发展表现出性别差异, 男孩在力量和体格的 技能上发展更好，女孩则在身体协调技能上发展更 好 $^{[17]}$.

手的使用和制作工具在人类种系发展中具有里程 碑式的意义, 同样地, 个体发展中手的动作发展是个体 心理发展的一个重要组成部分. 精细动作的发展使得 儿童可以够拿、书写、艺术创作以及生活自理等. 在 精细动作技能发展方面, 新生儿出生时已有抓握反射, 表现出够物倾向. 大约 3 个月时, 够拿动作准确性逐渐 提高, 但由于婴儿手眼协调能力有限, 尚不能抓住或拿 住客体. 4 5 个月婴儿会协调两只手来探索物体. 大多 数贞儿在 $5 \sim 6$ 个月时能有意识够拿、抓握和拿住各种 小物体, 并利用视觉提高抓握的准确性. 6 9个月时, 婴 儿只需伸出一只手便可够拿小物体. 8 11个月之间, 婴 儿的抓握和够物得到大量练习, 使其注意力从够物上 转移并分配到拿到物体之后需要完成的事情上 ${ }^{[18]}$. 随 着进一步的发展, 婴儿的手眼协调能力以及对小肌肉 的控制能力大大提升，这使得他们可以用手做更为复 杂的动作. 接近 1 岁时, 婴儿的钳形抓握能力开始出现, 这提升了贞儿完成基本生活动作的能力，基本生活动 作能力是儿童早期的重要发展任务之一, 主要包括的 技能有穿衣、洗漱和进食等. 1 1.5 岁时，婴儿开始使 用手抓握勺子自己吃饭, 但仍需成人的帮助. 2 岁左右 的幼儿还不能很好地完成穿衣、切食物和画画等较复 杂动作. 3岁的儿童可以穿脱简单的衣服、系纽扣、系 鞋带等. 到5岁时, 儿童能完成所有这些任务, 甚至可以 用剪刀剪出一条直线, 或用蜡笔书写字母和数字. 大部 分儿童在5.5岁发展出成熟的握笔姿势 ${ }^{[19]}$. 幼儿期精细 运动技能的表现还有一个非常吸引人的方面, 就是绘 画技能的发展. 2岁儿童的绘画处于涂鸦阶段, 儿童会 画一些垂线和折线; 3 岁左右的儿童处于形状阶段, 会 画出一些方形、圆形等形状; 3 4岁期间处于图案阶 
段，儿童会把形状组合成更复杂的图案. 4 5岁开始进 入图画阶段, 随着精细动作和认知的发展, 绘画中的现 实成分也逐渐增多.

根据动力系统理论, 婴儿的每一个新动作技能都 是为了实现重要目标而对既有技能的主动、复杂地重 新建构 ${ }^{[20]}$. 贞幼儿的动作发展过程中涉及到各种技能 的协调, 包括肌肉的发展、知觉能力和神经系统的发 展、执行特定动作的动机、周围环境的支持 ${ }^{[21]}$. 运动 技能的发展依靠复杂的系统, 各种技能相互协调相互 作用, 使贞幼儿可以去探索和控制外部世界.

\section{2 儿童青少年期的动作发展}

总体来看, 儿童青少年期动作技能的有效性、精 确性和稳定性均得以提高. 许多研究均已表明, 儿童 青少年期粗大动作的发展总体上随着年龄不断提高, 但不同类型粗大动作的发展速度存在差别, 表现出波 浪式增长的发展趋势 ${ }^{[7]}$. 10 12岁时, 粗大动作发展的 主要指标基本稳定并开始接近成人水平. 女孩的运动 发育似乎遵循不同于男孩的发育过程. Gidley Larson 等人 ${ }^{[22]}$ 对 144 名典型发育中的7 14岁儿童的运动系统 发育状况进行了评估, 结果发现, 女孩表现出的动作失 调都比男孩少, 而且比男孩更快、更熟练. 此外, 青少 年投掷时通常可以协调肩膀、手臂、躯干和腿部的力 量, 与年幼儿童相比, 把球扔得更远, 这不仅是由于他 们更高大和强壮, 更是因为他们可以运用更精确、更 有效的运动技巧 ${ }^{[23]}$. 青春期时男孩大肌肉活动的能力 继续增强, 而女孩则与以前持平或有所下降.

在精细动作的发展方面，儿童和青少年均表现出 随着年龄的增长而提高. 在儿童期, 精细动作的灵敏 性 $^{[24]}$ 、协调性 ${ }^{[25]}$ 存在男女性别差异. 对于儿童来说, 书写动作是一项重要发展任务以及能力要求, 完成书 写动作需要通过手部使用笔类等工具进行. 儿童学习 书写汉字时, 对其精细动作技能的要求更高. 除了手 部动作的发展外, 书写动作也是将感知觉与动作技能 协调的复杂过程, 书写过程的顺利完成需要一定水平 的整合能力. 1 3年级是手写能力快速发展时期 ${ }^{[26]}$, 真 正掌握书写技能的动作模式要到7岁. 青少年期在精细 动作的发展方面也有稳定的改善. 个体的手腕、手指 灵活能力以及双手协调能力的发展均随年龄的增长不 断提高, 且这一阶段精细动作发展不存在性别差异. 一 些特殊的患儿群体伴随着运动功能的损伤, 特别是手
部动作能力受损, 比如患有脑㿈的儿童和青少年经常 伴有手部功能受损, 这使得他们很难处理日常活动, 对这些特定群体的干预措施必须适合生活情况, 并融 入儿童和青少年的日常生活 ${ }^{[27]}$.

\section{3 成年期的动作发展}

成年早期/青年期(18 35岁)这一时期, 个体的身体 动作发展以及其他很多方面的发展都会达到巅峰状 态, 其中运动能力发展也处于高峰期. 许多运动技能在 25 35岁时达到巅峰. 以运动员群体为例, 对四肢的运 动速度、爆发力和协调要求较高的一些项目, 其竞技 成绩都在 20 岁左右达到巅峰; 对耐力、稳定性和瞄准 等能力要求高的项目, 其竞技成绩在 30 岁左右达到巅 峰 $^{[28 \sim 31]}$.

成年中期/中年期(35 60岁)这一时期，个体的生 理、心理都处于相对比较稳定的状态. 与其他发展阶 段相比, 这一时期个体生理及心理发展的最大特点就 是稳定与过渡并存. 个体的粗大动作和精细动作从成 年早期的巅峰状态后开始稳定发展, 并伴随着衰退. 大部分研究发现, 这种衰退不存在性别差异. 对于特 殊疾病导致运动障碍的个体而言, 成年中期也是比较 敏感的一个时期. 比如, 以脑痽患者为对象的研究发 现，相较于成年早期，成年中期患者粗大动作功能的 衰退在33 42岁的成年人中最为明显 ${ }^{[32,33]}$.

成年晚期/老年期(60岁之后)个体身心变化的总趋 势就是逐渐表现出退行性的变化 ${ }^{[2,34]}$. 衰老与各种认 知功能的衰退有关 ${ }^{[35]}$. 除了认知衰退, 老年人经常表 现出运动能力的衰退, 如精细运动控制能力降低、协 调困难、运动变异性增加、运动速度减慢、步态和平 衡等能力下降 ${ }^{[36 ~ 38]}$. 运动能力的衰退会影响老年人的 日常活动, 导致老年人活动受限、参与受限和死亡的 风险增加. 步态和平衡问题尤其令人担忧, 因为跌倒 是老年人受伤和发病的主要原因: $20 \% \sim 30 \%$ 的跌倒老 年人遭受中到严重伤害, 限制了其行动能力 ${ }^{[39]}$.

随着年龄的增长, 老年人在各种任务中的运动持 续时间明显增加，运动速度会减慢 $15 \%$ 30\%. 这种减 慢在某种程度上是因为老年人强调以运动速度为代价 的运动准确性 ${ }^{[40]}$. 老年人在双手和多关节运动的协调 性方面也存在缺陷. 例如, 当老年人同时移动他们的 肩关节和时关节, 而不是执行单一的关节动作时, 运 动变得缓慢和不流畅 ${ }^{[41]}$. 姿势稳定性(postural stabi- 
lity)也常常随着年龄的增长而降低 ${ }^{[37,42]}$. 姿势控制代 表了感觉和运动系统之间的复杂相互作用, 包括感知 环境刺激, 对环境中身体方向的改变做出反应, 并在 支撑的基础上保持身体的重心. 与年轻人相比, 老年 人在稳定姿势下的姿势摆动增加, 无法执行有效的步 进反应, 难以控制重心和压力中心相对于其稳定极限 的位移. 运动功能随着年龄的增长而下降, 导致这种 下降的原因是复杂和多因素的, 主要包括肌肉骨骼结 构、周围和中枢神经传导、本体感受、神经肌肉连接 等 $^{[43]}$.

体育活动对老年人认知能力的影响已经得到了大 量的验证. 不同的干预措施, 如有氧运动、力量和大肌 肉运动训练项目已被证明可以改善认知功能. 针对身 体健康和大肌肉运动技能的不同锻炼计划也会促进健 康老年人认知能力的改善 ${ }^{[44,45]}$.

总的来看, 动作的产生以及动作技能的获得是贞 儿阶段发育的里程碑，儿童青少年期的动作技能的有 效性、精确性和稳定性均得以提高, 许多运动技能表 现在青年期达到巅峰. 成年中期稳定发展, 并伴随着 衰退, 老年期出现明显的运动能力下降.

\section{2 动作发展的测评工具}

儿童的动作不仅是个体发展的重要组成部分, 对 个体心理发展具有不可忽视的促进作用, 而且也是人 们观察、评价儿童发展的重要手段. 从20世纪初开始, 动作发展就成为儿童早期发展评估的主要指标 ${ }^{[46]}$. 本 文主要汇总选取了两个类型: 发育能力评定量表(网络 版附表1)和专门的动作能力评定量表(网络版附表2), 共12种动作能力测量工具进行了总结.

国内外有关儿童早期发展的评估量表通常包括对 动作领域的考察, 能刻画出早期动作技能发展的水平. 这些测量工具通常用于初步检查, 并且许多评估量表 在临床具有很强的应用价值. 例如, 格赛尔发育诊断 量表(Gesell Developmental Schedules, GDS)的测验内 容中包括粗大运动行为和精细运动行为的测量, 其结 果可以作为发育迟缓和智力残疾诊断的重要依据. 贝 利婴幼儿发展量表(Bayley Scales of Infant and Toddler Development-Third Edition, BSID-III)的运动量表分为 粗大动作和精细动作, 运动领域得分低于临界值, 也表 明孩子可能在运动方面存在发育迟缓, 提示需要进一
步临床评估和干预 ${ }^{[47]}$. 麦卡锡幼儿智能评定量表 (McCarthy Scales of Children's Abilities, MSCA)的运 动分量表包括粗大动作平衡能力及精细动作的协调能 力测试. 该量表测试年龄范围较广, 测验内容的趣味性 较强, 适合对幼儿及智力发育障碍的儿童进行测量.

国内的发育评定量表主要有: 0 6岁儿童神经心理 发育量表、0 3岁婴幼儿发育量表(Child Developmental Center of China, CDCC) 以及3 6岁中国儿童发展量 表 ${ }^{[48,49]}$. 这些量表均包括儿童早期运动能力的诊断和 笁查, 填补了国内的空白, 对早期诊断和早期干预有 重要的参考意义. 需要注意的是, $\mathrm{CDCC}$ 对异常运动模 式儿童的识别具有一定的局限性, 也缺少农村常模, 使 用时需要注意.

除了常用的综合发育评估量表, 专门的运动能力 评估的量表也可用于儿童早期发展的评估. Peabody运 动发育量表(Peabody Developmental Motor Scales, PDMS)可用于6岁以下的正常儿童以及运动发育异常 儿童的运动发育水平的评估. 该量表还配套有针对量 表测试项目的教育和训练计划, 具有评估和干预的双 重作用 ${ }^{[50]}$. 粗大动作发展测试(Test of Gross Motor Development, TGMD) 除了包括动作能力的评估, 还结 合了动作质量 ${ }^{[51]}$. 儿童运动协调能力评估量表(Movement Assessment Battery for Children, M-ABC)对儿童 的精细运动、粗大动作和平衡能力的发育状况进行了 全面的评估, M-ABC-2根据测试者年龄分为 3 个阶段 进行不同内容的测试: 3 6岁、7 10岁、11 16岁. 该 量表在世界范围内广泛应用, 均表现出较好的信效 度 $^{[52]}$. 马氏灵敏运动技能测评(Maastrichtse Motoriek Test，MMT)在整体运动技能评分中包含了定性观察, 从而对儿童的运动能力提供了一个更全面的观点. 该 量表的一个限制是缺乏位移运动技能项目, 并且年龄 范围比较小 ${ }^{[53]}$. 布尼氏动作熟练度测试(BruininksOseretsky Test of Motor Proficiency, BOT)是目前国际 上广泛应用于智力障碍儿童动作发展的评估工具之 一. BOT-2 的测试年龄范围也较广, 可用来鉴别有轻度 到中度运动协调缺陷的个体 ${ }^{[54,55]}$.

评估整个生命周期内运动能力的变化是一项复杂 的测量任务. 精确测量运动功能和能力通常需要专门 的测试环境和工具. 在不同的研究和不同的年龄跨度 中, 运动功能的测量缺乏一致性, 这限制了研究结果 的普遍性. 由美国国家卫生研究院研发的NIH神经及 
行为功能评估工具 $(\mathrm{NIH}$ Toolbox for Assessment of Neurological and Behavioral Function)包含一套完整的 对动作、认知、感觉和情感功能的测试工具, 适用年 龄为3 85岁. 其中关于动作测评的内容包括 5 大部分: 灵巧性、强度(上肢、下肢)、平衡、位移运动和持久 力 $^{[34]}$.

\section{3 动作毕生发展的大脑基础}

认知神经学科和脑科学技术的革命性发展为研究 动作领域的毕生发展机制提供了基础和契机. 关于人 类和非人类灵长类动物的研究表明, 运动行为的大脑 基础包括主要运动区域(primary motor areas), 如初级 运动皮层(primary motor cortex, M1), 以及分布较广的 非主要运动区域(nonprimary motor areas), 包括体感皮 层(somatosensory cortex)、辅助运动区域(supplemen-

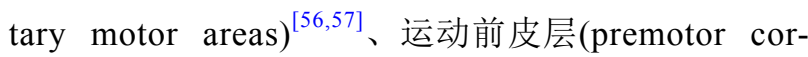

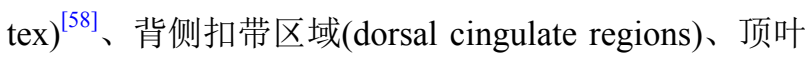
区域(parietal regions)以及小脑(cerebellum) ${ }^{[59,60]}$ 等. 这 些区域直接参与运动活动的执行以及对运动功能重要 的感知和认知 ${ }^{[61 \sim 65]}$.

有大量证据支持灵长类具有多个不同的动作模 块. 部分通路的模型示意图如图1所示. 背侧流对伸 够、抓握动作有独立的分区，传递视觉信息到M1。一 条路线连接了顶枕区域(parieto-occipital area, PO)和背 侧前运动皮层，这种连接有两者直接的联系及经过顶 内沟(上部顶叶模块)的间接联系. 这一系统对于直接 编码物体的动作(伸够)非常重要. 第二条路线从背侧 纹状皮层通过前顶内区域到腹侧前运动皮层，将物体 的内源性视觉属性转化为执行抓握的运动命令 ${ }^{[66,67]}$.

\section{1 婴幼儿期动作发展的大脑基础}

当婴儿出生时, 大脑皮质下层就已经发育完全, 它 负责调节大多数反射行为, 以及呼吸和心律等基本的 生命活动. 伴随着脑的不断发育，大脑皮质中的细胞 也越来越成熟，相互之间的联结也不断增多，这些细 胞将负责复杂的运动技能以及认知和语言等高级的思 维过程. 伴随着突触联结的增多, 大脑会出现髓鞘化 (myelinization). 在1岁之前, 大脑发育主要是神经纤维 髓鞘化，以及神经纤维不断延伸或生长为“树突树”的 过程. 有的神经元会运动到大脑的表层, 即大脑皮质 (cerebral cortex), 另一些神经元则运动到大脑皮质下 层. 这种在生命早期大脑中不断增加的髓鞘化影响精 细运动能力的发展. 婴儿从出生到 1 岁时存在几个运 动高峰期. 例如, 在 3 4 个月会出现一个动作高峰期, 此时，贞儿正自发地伸手去够物体; 到 8 个月大时，贞 儿学会爬, 并寻找感兴趣的物体; 到 12 个月大时, 贞儿 正学习走路. 这些动作的高峰期与突触之间联结的大 量形成有关. 当婴儿不断地重复并练习简单的感觉运 动模式(例如转头或伸手够物品)时, 他们也稳固了重 要的突触间的联结. 所以有趣的环境能提高贞幼儿的 活动水平, 如果鼓励他们积极地与周围的人和物进行 互动, 就可以提高贞儿大脑功能的质量(突触联结密 度)以及强化突触间的联结(髓鞘化增加) ${ }^{[68]}$.

2岁前婴儿的大脑网络结构基本上已经表现出成 人化的网络交互模式. 将新生儿大脑与成人大脑网络 结构进行比较发现，大脑网络结构在生命的前 2 年基 本均已出现. 在大多数高阶认知网络中, 新生儿表现 为孤立的局部连接性，而在出生后的第一年，分布的 网络结构在逐渐发育, 并且不同的网络也在建立越来 越成人化的网络交互模式. 与其他网络相比, 在感觉 运动网络中, 从新生儿到2岁, 这种交互模式表现为更 显著的网络层次关联性降低而不是增加的特点 ${ }^{[69]}$.

生命早期的大脑损伤与婴儿随后的运动能力是密 切相关的. 围产期大脑结构网络的损伤会预测 8 个月婴 儿的运动能力 ${ }^{[70]}$. 即使在没有解剖学损害的情况下, 极早产儿的负责运动、语言和执行功能的关键大脑网 络的功能连接在出生后不久就会出现缺陷 ${ }^{[71]}$. 对出生 到22个月婴儿进行的追踪研究也发现, 左楔前叶(left precuneus)、右枕上回(right superior occipital gyrus)、 右海马(right hippocampus)的神经特性可预测BSIDIII所测得的运动功能障碍 ${ }^{[72]}$.

功能网络水平的脑-行为关系的变化也是婴幼儿 期步行和粗大运动能力出现和巩固的基础. 关于贞儿 的大脑功能网络连接研究表明, 功能网络在贞儿期容 易识别, 并已开始表现出包括感觉运动网络在内的多 种网络的早期成熟特征 ${ }^{[73 \sim 75}$. 在生命早期, 运动能力 特别是粗大运动能力的出现和提高也与功能网络内和 功能网络间的连通性相关，具体表现为：在12个月时, 动作的大脑功能连接和默认模式网络(default mode networks)与行走相关; 然而, 在 24 个月时, 动作的大脑 功能连接与背侧注意(dorsal attention)和后扣带神经网 


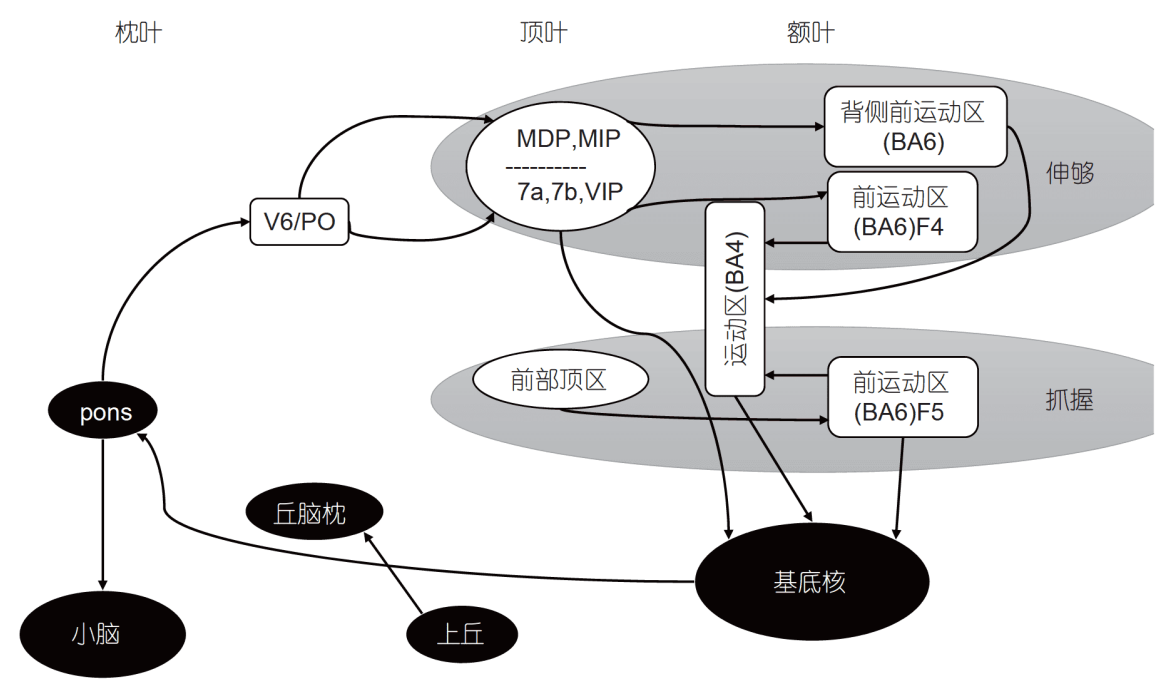

图 1 两个不同动作流所涉及的灵长类大脑区域及其连接示意图. BA: 布洛德曼区; BA6: 布洛德曼6区; F4, F5：额叶区域; MDP: 背内侧顶叶; MIP: 内侧顶区; PO: 顶枕区; V6: 视觉区6; VIP:腹侧顶叶; pons: 脑桥; 7a: 皮质区7a; 7b: 皮质区7b

Figure 1 The schematic diagram of the brain regions and connections of primates involved in two different motor flows. BA: Brodmann area; BA6: Brodmann area 6; F4, F5: frontal areas; MDP: mediodorsal parietal; MIP: medial intraparietal; PO: parietal-occipital; V6: visual area 6; VIP: ventral intraparietal area; 7a: cortical area 7a; $7 \mathrm{~b}$ : cortical area $7 \mathrm{~b}$

络(posterior cingulo-opercular networks)相关. 对粗大动 作的功能分析也显示, 在 12 和 24 个月时有运动和默认 模式网络的参与，在 24 个月时有背侧注意、扣带回、 额顶骨和皮质下网络的额外参与 ${ }^{[76]}$.

\section{2 儿童青少年期动作发展的大脑基础}

儿童早期精细动作发展与神经系统的成熟密切相 关, 个体的动作经验也会促进神经系统的成熟 ${ }^{[6]}$. 贞儿 出生后, 随着年龄增长, 具体的经验使得突触及灰质的 数量出现修剪且伴随着灰质数量的下降, 进而出现白 质数量的增加. 白质在感觉运动区增加得较早, 这使 得儿童早期的精细动作能力出现提升 ${ }^{[77,78]}$.

胼胝体运动纤维和双侧皮质脊髓束中的白质微结 构是两个众所周知的白质通路，对粗大和精细运动功 能都至关重要 ${ }^{[79,80]}$. Grohs等人 ${ }^{[81]}$ 利用弥散张量成像 (diffusion tensor imaging, DTI)描绘了儿童期的动作发 展路径, 并描述了其与 80 名3 6岁的典型学龄前儿童的 运动表现之间的关系, 结果发现, 具有较好运动能力的 学龄前儿童在动作发展路径中显示出更成熟的白质模 式, 主要表现为皮质脊髓束(corticospinal tracts)和局部 区域内较高的分数各向异性(fraction anisotropy, FA)以 及较低的平均扩散率(mean diffusion, MD) 和径向扩散 率(radial diffusion, RD), 与更好的运动表现有关. 这种
白质的不同成熟模式也表现在具有特定运动专长的个 体(即音乐家、高尔夫球手和空手道黑带)与缺乏专长 的年龄匹配对照组研究中, 具有特定运动专长的个体 在运动白质脊髓束中具有更高的FA和更低的扩散

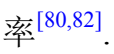

小脑在感觉运动和认知功能中发挥重要作用, 自 闭症谱系障碍(autistic spectrum disorder, ASD)患者小 脑的细胞、体积和功能均有异常 ${ }^{[83]}$. 对儿童精细动作 的研究表明, 除其他大脑结构外, 顶叶皮层(parietal cortex)和小脑(cerebellum)也参与其中 ${ }^{[84]}$. 小脑被认为 是精细运动的控制中枢, 参与动作的预测, 对于动作 表现和动作过程至关重要, 而顶叶皮层与小脑这两种 结构与心理表征和运动表象也密切相关. 顶叶皮层的 发育、动作表征和形成与运动表象相关的内部模型能 力之间有密切联系. 随着年龄的增长, 顶叶皮层的成熟 过程提高了神经效率, 并可能使年龄较大的儿童(10岁 以上)逐步提高他们产生准确运动预测的能力 ${ }^{[85]}$.

前额叶是多种认知功能的大脑基础, 此外, 在运动 技能学习中, 前额叶也发挥很关键的作用 ${ }^{[86]}$. 针对大 脑皮层的研究发现，在精细运动过程中，除运动区、 感觉区等大脑皮层激活外，前额叶也同时被激活 ${ }^{[87]}$. 动作干预训练也会促进额叶发育. 有研究发现, 接受 8 个月有氧运动干预的儿童大脑的默认模式(default 
mode)、认知控制(cognitive control)和运动网络(motor networks)表现出更多的空间精细化, 并且与右侧额叶 内侧回(right medial frontal gyrus)的运动网络同步性 更强 ${ }^{[9]}$. 运动会影响大脑皮层的体积, 研究表明, 额 叶、顶叶和颢叶皮质的特定大脑区域容易受到运动的 影响，这可以通过整个生命周期以及不同健康人群内 的皮质体积和皮质厚度的变化来表示 ${ }^{[88,89]}$.

除了大脑结构, 动作控制还依赖于完善的动作环 路, 这对儿童的动作发展至关重要. 在年长儿童和成 人中, 运动网络内的功能连接以及网络间的连通性与 运动表现有关 ${ }^{[65,90]}$, 功能连接的区域间和区域内改变 与孤独症儿童的运动缺陷有关. 从儿童早期到青少年 晚期, 初级视觉、听觉和感觉运动系统存在共同的网 络轨迹模式, 这些网络是在儿童早期建立的, 在青春 期早期经历了显著的扩张, 但在青春期后期收缩或修 剪. 也就是说, 5 8岁儿童早期的初级运动皮质(右中 央前回)(right precentral gyrus)与左中央前回(left precentral gyrus)、双侧中央后回(bilateral postcentral gyri) 和内侧辅助运动皮质 (medial supplementary motor cortex)相关, 儿童晚期(8.5 11岁)到青少年晚期(16 18岁) 的初级运动皮质(右中央前回)网络连接范围持续存在, 但与青少年早期相比, 青少年晚期的网络范围相对减 少 ${ }^{[91]}$. 而且优越的运动发育与内在运动网络连接中建 立左半球优势密切相关, 运动能力表现好的儿童的左 半球运动回路连通性更好, 左侧壳核-丘脑网络连接、 左侧辅助运动区-主要运动区连接以及左侧辅助运动 区-小脑前连接分别与运动速度、运动节律和运动控 制显示出高相关性 ${ }^{[65]}$.

大量研究表明, 儿童时期的动作技能对认知功能 具有积极作用 ${ }^{[92,93]}$. 许多元分析研究发现, 体育活动 对儿童的某些认知功能(如语言学习、学术成就、注 意力和工作记忆)均会产生有益的影响 ${ }^{[94-98]}$. 一项关于 运动对青少年影响的综述表明, 运动有益于典型发育 中儿童的白质结构的完整. 此外, 运动对患者人群(如 脑瘤康复的患者)的白质结构(例如完整性)也有影 响 ${ }^{[99,100]}$.

越来越多的证据都表明, 认知和运动功能是相互 关联的, 可能依赖于相同的皮层和皮层下神经结构的 发展 ${ }^{[101]}$, 动作的发展与认知的发展共享部分脑区 ${ }^{[8]}$, 前额叶和后顶叶的协调与感觉运动区域的活动有关. 所以了解动作发展的大脑机制有助于更深刻地理解动
作与认知发展的关系, 从而促进认知及个性和社会性 的健康发展.

\section{3 成年期动作发展的大脑基础}

成年早期是许多运动能力发展的巅峰时期. 运动 员群体作为动作发展和技能学习的一个特殊群体和典 型群体, 这一年龄阶段的运动员与普通对照组相比, 无 论是大脑结构还是大脑功能都存在较大的差异, 这种 差异正是运动训练导致的大脑可塑性的变化 ${ }^{[102,103]}$. 相比于普通对照组, 羽毛球运动员群体的小脑灰质体 积增大，并且小脑不同亚区的静息态激活减少，但小 脑亚区域之间以及小脑亚区域和大脑皮质(如中扣带 皮层和海马体)之间的功能连接增加 ${ }^{[104,105]}$. 羽毛球及 篮球运动员等小球类运动员的左侧前扣带回、脑岛、 丘脑和小脑蚓部脑区也存在更有效的自主神经反应环 路 ${ }^{[106,107]}$. 与普通对照组相比, 跳水运动员在感觉运动 区与运动前区表现出大脑灰质体积的变化, 并且长期 的跳水训练可以通过突触修剪改善神经回路, 这可能 是他们非凡潜水表现的解剖学基础 ${ }^{[108,109]}$. 枕顶叶、 前扣带回和尾状核的灰质体积变化表现在马拉松运动 员群体中 ${ }^{[110,111]}$. 优秀空手道运动员的题下/上(inferior/ superior temporal)、枕叶(occipital)、前运动皮层和颢 极上(temporal pole superior)的灰质体积增加, 同时, 精英空手道运动员的运动计划和视觉感知的大脑区域 也被发现具有较高的连接性 ${ }^{[112]}$.

与老年人(older adults)相比, 成年人 (younger adults)的前运动区域和M1之间的功能和结构连接性 的差异性与个体的运动能力表现相关, 这些区域之间 的连接性越强, 反应时间就越快 ${ }^{[64,113,114]}$. 在动作选择 过程中, 大脑功能连接与白质连接区域的局部区域(包 括前运动区和运动皮质) 的FA有高度特异性的相 关 ${ }^{[113]}$. 那些在动作完成过程中表现出更强时间精确性 的个体, 在右半球的中央前回(precentral gyrus)、中枕 回(middle occipital gyrus)、壳核(putamen)以及左半球 的丘脑(thalamus)、楔叶(cuneus)和小脑(cerebellum)的 激活会明显增强 ${ }^{[115]}$.

在整个成年期, 对于动作发展的研究更多地集中 在老年期群体. 大多数关于老年期运动能力的研究都 一致认为, 衰老与运动能力和运动协调能力的下降是 密切相关的 ${ }^{[37,116]}$, 这也是生理衰老的一个重要方面. 老年人关于运动的主要神经表征和潜在的补偿性认知 
机制似乎都受到破坏. 随着年龄的增长, 运动能力的损 害可能部分是由于感觉受体、肌肉、周围神经、关节 等周围结构以及中枢神经系统的改变. 过去的研究主 要集中在外周机制, 但近年来的研究重点已转移到更 为核心的机制上. 正常的衰老通常也伴随着大脑功能 和结构的改变. 研究者发现, 运动网络内的功能连接 以及网络间的连通性、连通强度与运动表现有 关 ${ }^{[90,117]}$. 衰老伴随着感觉运动网络中静息状态功能连 接的减少 ${ }^{[118]}$. 与年龄相关的运动表现下降也可能是白 质束退化的结果, 这也会影响大脑结构连接. 除了结构 连接, 衰老与大脑的功能连接也密切相关. 与年轻人相 比，老年人的感觉运动皮层中后侧脑岛功能整合随着 年龄的增加而减少，这种降低的静息态功能连接可以 预测老年人的老化 ${ }^{[119]}$.

与年龄相关的运动皮质区和胼胝体萎缩可能会加 速运动能力的下降，如平衡、步态缺陷、协调缺陷和 运动减慢. 与此同时, 神经递质系统的退化(主要是多 巴胺系统)可能与年龄相关的粗大动作和精细动作的 退化与高级认知系统退化均有关. 老年人在运动表现 期间通常表现出比年轻人更强的区域性脑活动，也表 现出更广泛的大脑运动控制区域参与，特别是前额叶 皮质和基底神经节网络，这些区域对年龄效应很敏 感 ${ }^{[37]}$. 在针对帕金森患者的研究中, 病人会表现出前 额叶和枕叶灰质明显地减少，行为上表现为精细动作 速度减缓，说明精细动作速度与前额皮层的灰质密切 相关 ${ }^{[120]}$.

但是, 也有研究者持不同的观点, 认为随着年龄的 增长，前额叶皮质对运动老化反而会表现出补偿作用. 与年龄相关的运动减缓伴随着区域激活和有效功能连 接的增加, 特别是针对初级运动皮层区域的运动耦合. 老年组中的年轻参与者表现出初级运动皮层的强连接 性, 这一连接性随着年龄的增长而稳步下降. 相反地, 随着年龄的增长, 前额叶对运动系统的影响增加. 在衰 老过程中，在前额叶-前运动皮层-初级运动皮层轴中 更高的耦合预示着更快的心理运动速度. 因此，在核 心运动网络中，前额叶皮层的重要作用是代偿减少的 耦合 ${ }^{[36]}$. 随着年龄的增长，运动皮层和小脑与壳核的 连通性增强，这就证明了随着年龄的增长，跨网络的 互动性更强，而这种更大的网络相互作用也可补偿运 动能力随着年龄下降 ${ }^{[90]}$. 越来越多的证据表明了身体 锻炼对延缓认知老化方面的作用. 身体锻炼通过对某
些大脑结构的重塑来影响认知老化 ${ }^{[121,122]}$, 经常参加 体育锻炼对于老年人维持大脑灰质和白质容量、白质 完整性等方面均表现出积极作用 ${ }^{[123,124]}$. 最近一项关于 24 76岁的健康和被诊断出患有临床疾病的参与者的 元分析结果表明，各种类型的运动可能会减轻与年龄 相关的左海马体积损失 ${ }^{[125]}$.

综上所述, 从毕生发展的角度, 已有的研究定位了 与动作发展相关的大脑区域和大脑网络，探究了动作 的产生、发展、衰退的大脑结构变化及大脑网络连接 的改变. 运动对个体认知发展以及个性和社会性发展 产生积极影响，大脑可塑性在其中起到的作用，以及 更复杂的运动技能掌握背后的神经机制还有待进一步 探究.

\section{4 学科未来发展方向的预测和展望}

动作技能的获取既是发展的基础，又是促进发展 的催化剂，它与感知、认知、情感、社会互动和社会 认知紧密相关. 目前在动作认知神经发展研究领域虽 然取得了一些成就，但依旧面临着很多的挑战，也面 临着很大的发展契机. 基于目前的研究现状，本文总 结出今后发展的趋势可能主要会有以下 5 个方面.

(1) 学科领域整合化. 在运动及动作发展领域的 多学科整合以及交叉研究的趋势会越来越强. 认知科 学、运动心理学、发展心理学、神经科学以及生物科 学的学科整合形成新的交叉研究领域. 动作及运动发 展虽然依托发展心理学这门学科, 在新的学科交叉发 展的背景下，依靠这种学科交叉、协同多个发展主题 (如认知发展、情绪和社会性发展等)来实现创新是该 领域可持续发展的重要方向. 这种新的研究视角将对 运动认知发展或动作认知发展进行更客观的科学 解答.

（2）研究视角积极化. 未来关于运动认知领域的 研究会更多地采取积极视角, 更关注正常群体的积极 发展. 动作技能的掌握和健康发展与人的毕生健康发 展呈现密切的关联性. 目前可能更多关注的是非正常 群体的运动康复和干预，未来的研究要更多关注健康 群体的运动预防及更积极的促进作用. 比如，目前融 合了正念冥想的新兴的、有中国传统特色的有氧活动 模式对轻度认知障碍及产后抑郁等的干预治疗已经初 见成效. 
(3) 研究方向精细化. 运动认知神经发展领域的 未来研究越来越精细化, 主题会更加聚焦. 该领域经历 了研究方向从无到有, 研究群体由单一到多元, 研究主 题从分散到聚焦的过程. 国内的研究团队以1989 2019 年 30 年为样本，按照 10 年为一个节点对所有关于运动 的文献做了汇总及梳理. 可以很明显地发现，该领域 的研究趋势在第一个10年(1989 1999年)的主题是相 对分散的，聚焦点不多; 第二个10年(2000 2009年)聚 焦点明显增多, 个别主题词的权重增加; 近十年 (2010 2019年)的研究趋势主题非常聚集, 主题词的外 延更加扩大，包含的内容更丰富. 魏高峡等人 ${ }^{[126]}$ 综述 分析了基于磁共振成像技术的运动员可塑性的研究, 并总结了其发展态势, 结果发现, 运动员大脑磁共振成 像的相关研究的研究热点、技术手段和主题随着时间 出现了一定的演化, 以运动专家知识系统作为研究主 题的热点仍然延续, 但多侧重于对动作预期、动作控 制的研究选题.

(4) 研究范围纵向化. 更大年龄范围的纵向研究 以及大数据背景下的分析势在必行. 运动、动作和大 脑发育密切相关是一个常识, 研究者需要提供确業的 证据说明运动与大脑相关结构和功能的更深层的神经 机制. 小样本存在很多局限性, 要通过大数据大样本来 揭示研究结果的普遍性, 而大样本的获得就需要脑科 学的科普推广和新研究工具的突破. 此外, 拓展纵向 追踪研究及在实践中的应用训练研究也是未来的研究 方向和趋势.

(5) 研究工具多样化. 新的研究工具和研究技术 的诞生和革新发展会为脑发育研究提供新的突破, 为 更客观的科学解释提供可能. 目前最常用的影像检查 工具, 如功能磁共振成像(functional magnetic resonance imaging, fMRI)、事件相关电位(event related potential, ERP)、功能性近红外光谱技术(functional near-infrared spectroscopy, fNIRS)、经㐿磁刺激(transcranial magnetic stimulation, TMS)等的应用虽然已经比较广泛和 普遍, 但仍存在很多局限性. 未来更好的脑成像工具的 革新(更适合老年人、贞儿等特殊群体)，会极大促进 该领域的发展. 比如，生态学的便携式的设备可以更 自由地监控大脑的活动, 可能会颠覆目前的一些研究 发现，也会促使一些新的研究热点出现. 虚拟现实技 术、多模态处理以及时间/空间同时采用等各种技术 整合联动, 根据研究任务, 综合运用各种研究方法及技
术手段, 可以弥补单一方法的缺陷.

\section{5 国内发展路线图}

对比在动作发展领域的认知发展神经科学视角的 国内外研究, 会发现二者在研究主题、应用转化和专 业团队及平台搭建方面存在较大的差距. 我国已经将 “脑科学与类脑研究”上升为国家战略. 2017年, 我国 全面启动“科技创新2030一重大项目”, 这其中就包含 “脑科学与类脑研究”, 在此大背景下, 国内关于动作 及运动发展神经科学领域的发展路线应着重以下 3 方面.

(1) 重视领域、学科之间的结合. 运动发展的研 究将会更多地受到生物学(如动力学系统)、计算神经 科学、临床科学(将运动表现和可塑性作为诊断工具 和治疗)以及计算机科学(机器人学和人工智能)等领域 的启发. 目前正在发展中的机器人学已经在尝试理解 适应性、可生存行为. 应该把这些趋势看作是一种契 机, 对动作及运动技能发展的研究有可能激发一门具 身的、嵌入式的发展科学.

(2) 重视应用转化和干预研究. 动作的发展对于 生命早期的认知发展的重要性已经被广泛验证. 从毕 生发展的角度来看, 动作及运动的发展研究对于随后 的幼儿期、儿童青少年期以及现在普遍关注的老年 期这些生命发展的不同阶段的意义都越来越受到重 视. 运动对认知的促进作用也是目前国内和国外均关 注的焦点. 在教育领域, 很早就开始探查孩子的脑发 育状况、体育课程设置、运动锻炼与更高阶的认知 能力之间的关系. 比如对肥胖儿童的体育训练, 对阅 读障碍或注意力障碍等儿童群体的干预. 国际上的最 新研究确定了贞儿期及儿童期大脑良好发育的指标 和发展路径, 这其中也包括与动作及运动密切相关的 脑结构、脑功能及脑网络连接的发展. 在运动行为与 大脑可塑性的发展主题下, 运动作为一种干预手段可 以提升个体的自我调控功能. 鉴于目前全球面临的老 龄化问题, 国内外的另一个研究热点就是正常年老化 的老年群体以及轻度认知障碍群体(介于正常老化和 阿尔兹海默症之间)的身体活动或者运动锻炼对认知 功能老化的补偿和延缓功能背后的神经机制, 以及各 种运动干预是否能达到临床治疗的效果. 相比于药物 治疗或干预的副作用, 这也是运动干预的鬼力和价值 
所在.

（3）积极搭建研究平台及团队. 国外在运动及动 作的认知神经发展方面的研究机构设置是相对独立 的，也是相对完善的. 而在国内, 关于运动领域的相关 专业和学科更多地是被包含在体育学院, 更多地以运 动员群体为研究对象并服务于运动员群体. 2019年, 中国心理学会成立了运动认知神经科学学组, 初步搭
建起运动领域的认知神经研究的学术平台，该平台汇 集了运动领域的各个子方向的研究者, 诸如运动员群 体研究、儿童青少年运动技能学习研究、老年人认知 老化的运动干预研究; 还有诸如基础研究方向的运动 学习、运动控制、运动决策等. 目前在国内的运动或 动作认知神经发展的研究领域，平台和研究队伍的全 面建设势在必行.

\section{参考文献}

1 Gibson E J, Pick A D. An Ecological Approach to Perceptual Learning and Development. New York: Oxford University Press, 2000

2 Lin C D. The Developmental Psychology (in Chinese). 3rd ed. Beijing: People's Education Press, 2018 [林崇德. 发展心理学. 第3版. 人民教育 出版社, 2018]

3 Campos J J, Anderson D I, Barbu-Roth M A, et al. Travel broadens the mind. Infancy, 2000, 1: 149-219

4 von Hofsten C. An action perspective on motor development. Trends Cogn Sci, 2004, 8: 266-272

5 Dong Q, Tao S, Zeng Q, et al. The role of action in individual's early psychological development (in Chinese). J Beijing Norm Univ (Soc Sci), 1997, 4: 48-55 [董奇, 陶沙, 曾琦, 等. 论动作在个体早期心理发展中的作用. 北京师范大学学报, 1997, 4: 48-55]

6 Li H, He L. The relations between locomotion and cognition in early child development (in Chinese). Adv Psychol Sci, 2003, 11: 315-320 [李 红, 何否. 儿童早期的动作发展对认知发展的作用. 心理科学进展, 2003, 11: 315-320]

7 Zhu D P. The theory, method, measurement and development of the research on children's motor skill (in Chinese). J Nanjing Inst Phys Educ (Natural Science), 2015, 14: 15-21 [祝大鹏. 儿童动作发展的理论、方法、测评与发展趋势. 南京体育学院学报, 2015, 14: 15-21]

8 Diamond A. Close interrelation of motor development and cognitive development and of the cerebellum and prefrontal cortex. Child Dev, 2000, 71: 44-56

9 Krafft C E, Pierce J E, Schwarz N F, et al. An eight month randomized controlled exercise intervention alters resting state synchrony in overweight children. Neuroscience, 2014, 256: 445-455

10 Erikson E H. Childhood and Society. 2nd ed. New York: Norton, 1959

11 Erikson E H. Identity, Youth and Crisis. New York: Norton, 1968

12 Piek J P, Dawson L, Smith L M, et al. The role of early fine and gross motor development on later motor and cognitive ability. Hum Movement Sci, 2008, 27: 668-681

13 Murray G K, Veijola J, Moilanen K, et al. Infant motor development is associated with adult cognitive categorisation in a longitudinal birth cohort study. J Child Psychol Psychiat, 2006, 47: 25-29

14 Sigurdsson E, Van Os J, Fombonne E. Are impaired childhood motor skills a risk factor for adolescent anxiety? Results from the 1958 U.K. birth cohort and the national child development study. Am J Psychiatry, 2002, 159: 1044-1046

15 Fan C R, Zhou Z F. Study on the rule of intelligent development of children from birth to six years old (in Chinese). Acta Psychol Sin, 1983, 15: 62-77 [范存仁, 周志芳. 从初生到六岁儿童智能发展规律的探讨. 心理学报, 1983, 15: 62-77]

16 Gallahue D L, Ozmun J C. Understanding Motor Development: Infants, Children, Adolescents, Adults. New York: McGraw-Hill, 2001

17 Lung F W, Chiang T L, Lin S J, et al. Gender differences of children's developmental trajectory from 6 to 60 months in the Taiwan Birth Cohort Pilot Study. Res Dev Disabil, 2011, 32: 100-106

18 Claxton L J, Keen R, McCarty M E. Evidence of motor planning in infant reaching behavior. Psychol Sci, 2003, 14: 354-356

19 Wang X, Guo L P. A developmental study of pencil grasps of preschool children (in Chinese). Psychol Sci, 2009, 32: $579-583$ [王希, 郭力平. 学龄前儿童握笔姿势的发展性研究. 心理科学, 2009, 32: 579-583]

20 Thelen E. Motor development: A new synthesis. Am Psychol, 1995, 50: 79-95

21 Thelen E, Bates E. Connectionism and dynamic systems: are they really different? Dev Sci, 2003, 6: 378-391

22 Gidley Larson J C, Mostofsky S H, Goldberg M C, et al. Effects of gender and age on motor exam in typically developing children. Dev Neuropsychol, 2007, 32: 543-562 
23 Gallahue D L. Understanding Motor Development: Infants, Children, Adolescents. 2nd ed. Indianapolis: Benchmark Press, 1989

24 Chai J, Yang T L, Jiang S. Sensitivity study of children aged 7-12 under open situation-Test of snatching at six corner ball (in Chinese). J Shandong Inst Phys Educ Sports, 2011, 27: 60-65 [柴娇, 杨铁黎, 姜山. 开放情境下7-12岁儿童动作灵敏性发展的研究一六角反应球抓球 测试. 山东体育学院学报, 2011, 27: 60-65]

25 Pan T T. The research on gender difference of children's movement coordination ability between 7-12 years old (in Chinese). China Sport Sci Technol, 2002, 38: 15-17 [潘泰陶. 7-12岁儿童动作协调能力性别差异的研究. 中国体育科技, 2002, 38: 15-17]

26 Ren T T. Research on the methods and application of handwriting movement analysis in children (in Chinese). Dissertation for Doctoral Degree. Hefei: University of Science and Technology of China, 2017 [任亭亭. 儿童手写运动分析方法及其应用研究. 博士学位论文. 合肥: 中国科学 技术大学, 2017]

27 Lidman G, Himmelmann K, Peny-Dahlstrand M. Managing to learn bimanual activities - experiences from children and adolescents with cerebral palsy - a qualitative analysis. Disabil Rehabil, 2020, 1-9

28 Schulz R, Curnow C. Peak performance and age among superathletes: track and field, swimming, baseball, tennis, and golf. J Gerontol, 1988, 43: P113-P120

29 Lepers R, Cattagni T. Do older athletes reach limits in their performance during marathon running? Age, 2012, 34: 773-781

30 Zingg M, Knechtle B, Rüst C A, et al. Age and gender difference in non-drafting ultra-endurance cycling performance- the 'Swiss Cycling Marathon'. Extrem Physiol Med, 2013, 2: 18

31 König S, Valeri F, Wild S, et al. Change of the age and performance of swimmers across World Championships and Olympic Games finals from 1992 to 2013-a cross-sectional data analysis. SpringerPlus, 2014, 3: 652

32 Usuba K, Oddson B, Gauthier A, et al. Changes in gross motor function and health-related quality of life in adults with cerebral palsy: an 8-year follow-up study. Arch Phys Med Rehabil, 2014, 95: 2071-2077.e1

33 Jahnsen R, Villien L, Egeland T, et al. Locomotion skills in adults with cerebral palsy. Clin Rehabil, 2004, 18: 309-316

34 Reuben D B, Magasi S, McCreath H E, et al. Motor assessment using the NIH Toolbox. Neurology, 2013, 80: S65-S75

35 Grady C. The cognitive neuroscience of ageing. Nat Rev Neurosci, 2012, 13: 491-505

36 Michely J, Volz L J, Hoffstaedter F, et al. Network connectivity of motor control in the ageing brain. Neuroimage Clin, 2018, 18: 443-455

37 Seidler R D, Bernard J A, Burutolu T B, et al. Motor control and aging: Links to age-related brain structural, functional, and biochemical effects. Neurosci Biobehav Rev, 2010, 34: 721-733

38 Salthouse T A. Aging and measures of processing speed. Biol Psychol, 2000, 54: 35-54

39 Alexander B H, Rivara F P, Wolf M E. The cost and frequency of hospitalization for fall-related injuries in older adults. Am J Public Health, 1992, 82: 1020-1023

40 Seidler-Dobrin R D, He J, Stelmach G E. Coactivation to reduce variability in the elderly. Motor Control, 1998, 2: 314-330

41 Seidler R D, Alberts J L, Stelmach G E. Changes in multi-joint performance with age. Motor Control, 2002, 6: 19-31

42 Shkuratova N, Morris M E, Huxham F. Effects of age on balance control during walking. Arch Phys Med Rehabil, 2004, 85: 582-588

43 Ward N S. Compensatory mechanisms in the aging motor system. Ageing Res Rev, 2006, 5: 239-254

44 Berryman N, Bherer L, Nadeau S, et al. Multiple roads lead to Rome: combined high-intensity aerobic and strength training vs. gross motor activities leads to equivalent improvement in executive functions in a cohort of healthy older adults. Age, 2014, 36: 9710

45 Gajewski P D, Falkenstein M. Physical activity and neurocognitive functioning in aging - a condensed updated review. Eur Rev Aging Phys Act, 2016, 13: 1

46 Holsbeeke L, Ketelaar M, Schoemaker M M, et al. Capacity, capability, and performance: different constructs or three of a kind? Arch Phys Med Rehabil, 2009, 90: 849-855

47 de Onis M, Onyango A W, Borghi E, et al. Development of a WHO growth reference for school-aged children and adolescents. Bull World Health Organ, 2007, 85: 660-667

48 Fan C R. CDCC Infant Intelligence Development Test Manual (in Chinese). Beijing: Unity Press, 1988 [范存仁. CDCC婴幼儿智能发育测验 手册. 北京: 团结出版社, 1988]

49 Zhou R, Zhang H C. Introduction to CDCC children's development scale of China (in Chinese). Psychol Sci, 1994, 17: 137-140 [周容, 张厚粲. CDCC中国儿童发展量表(3-6岁)的编制. 心理科学, 1994, 17: 137-140]

50 Wu S K, Jiang G P. Research progress of assessment on young children's motor development (in Chinese). J Beijing Univ Phys Educ, 2014, 37 : 
81-87 [吴升扣, 姜桂萍. 儿童早期动作发展测量的研究进展. 北京体育大学学报, 2014, 37: 81-87]

51 Li J, Liang G L. Testing on big muscle group development (in Chinese). China Sport Sci Technol, 2005, 41: 105-107 [李静, 梁国力. 大肌肉群 发展测试(TGMD-2)研究. 中国体育科技, 2005, 41: 105-107]

52 Schulz J, Henderson S E, Sugden D A, et al. Structural validity of the Movement ABC-2 test: Factor structure comparisons across three age groups. Res Dev Disabil, 2011, 32: 1361-1369

53 Li B, Liu Y, Chen S T, et al. Introduction and implications of assessment tools for fundamental movement skills in children and adolescents (in Chinese). J Shanghai Univ Sport, 2018, 42: 8-28 [李博, 刘阳, 陈思同, 等. 儿童青少年基本运动技能测评工具研究及启示. 上海体育学院学 报, 2018, 42: 8-28]

54 Cools W, Martelaer K, Samaey C, et al. Movement skill assessment of typically developing preschool children: a review of seven movement skill assessment tools. J Sports Sci Med, 2009, 8: 154-168

55 Yuan Y Q, Liu Y, Ding J N. Application of Bruininks-Oseretsky Test of Motor Proficiency-Second Edition in assessing motor development among children with intellectual disability and its enlightenment to China (in Chinese). China Sport Sci Technol, 2019, 55: 14-20 [原雅青, 刘 洋, 丁佳宁. 布尼氏动作熟练度测试(BOT-2)在智力障碍儿童动作发展评估中的应用及对我国的启示. 中国体育科技, 2019, 55: 14-20]

56 Akkal D, Dum R P, Strick P L. Supplementary motor area and presupplementary motor area: Targets of basal ganglia and cerebellar output. J Neurosci, 2007, 27: 10659-10673

57 Mostofsky S H, Simmonds D J. Response inhibition and response selection: Two sides of the same coin. J Cogn Neurosci, 2008, 20: 751-761

58 Debaere F, Wenderoth N, Sunaert S, et al. Cerebellar and premotor function in bimanual coordination: parametric neural responses to spatiotemporal complexity and cycling frequency. NeuroImage, 2004, 21: 1416-1427

59 Konczak J, Timmann D. The effect of damage to the cerebellum on sensorimotor and cognitive function in children and adolescents. Neurosci Biobehav Rev, 2007, 31: 1101-1113

60 Lisberger S G. Visual guidance of smooth-pursuit eye movements: sensation, action, and what happens in between. Neuron, 2010, 66: 477-491

61 Fink G R, Frackowiak R S J, Pietrzyk U, et al. Multiple nonprimary motor areas in the human cortex. J Neurophysiol, 1997, 77: 2164-2174

62 Hanakawa T, Immisch I, Toma K, et al. Functional properties of brain areas associated with motor execution and imagery. J Neurophysiol, 2003, 89: $989-1002$

63 Rizzolatti G, Luppino G. The cortical motor system. Neuron, 2001, 31: 889-901

64 Lehmann N, Tolentino-Castro J W, Kaminski E, et al. Interindividual differences in gray and white matter properties are associated with early complex motor skill acquisition. Hum Brain Mapp, 2019, 40: 4316-4330

65 Barber A D, Srinivasan P, Joel S E, et al. Motor "dexterity"? Evidence that left hemisphere lateralization of motor circuit connectivity is associated with better motor performance in children. Cereb Cortex, 2012, 22: 51-59

66 Milner A D, Goodale M A. The Visual Brain in Action. New York: Oxford University Press, 2006

67 Jeannerod M. The Cognitive Neuroscience of Action. Trends Cogn Sci, 1997, 1: 238

68 Leonard H C, Hill E L. Review: The impact of motor development on typical and atypical social cognition and language: a systematic review. Child Adolesc Ment Health, 2014, doi: 10.1111/camh.12055

69 Gao W, Alcauter S, Smith J K, et al. Development of human brain cortical network architecture during infancy. Brain Struct Funct, 2015, 220: $1173-1186$

70 Linke A C, Wild C, Zubiaurre-Elorza L, et al. Disruption to functional networks in neonates with perinatal brain injury predicts motor skills at 8 months. Neuroimage Clin, 2018, 18: 399-406

71 Gozdas E, Parikh N A, Merhar S L, et al. Altered functional network connectivity in preterm infants: antecedents of cognitive and motor impairments? Brain Struct Funct, 2018, 223: 3665-3680

72 Schadl K, Vassar R, Cahill-Rowley K, et al. Prediction of cognitive and motor development in preterm children using exhaustive feature selection and cross-validation of near-term white matter microstructure. Neuroimage Clin, 2018, 17: 667-679

73 Fransson P, Aden U, Blennow M, et al. The functional architecture of the infant brain as revealed by resting-state fMRI. Cereb Cortex, 2011, 21: $145-154$

74 Gao W, Alcauter S, Elton A, et al. Functional network development during the first year: relative sequence and socioeconomic correlations. Cereb Cortex, 2015, 25: 2919-2928

75 Smyser C D, Snyder A Z, Neil J J. Functional connectivity MRI in infants: Exploration of the functional organization of the developing brain. 
NeuroImage, 2011, 56: 1437-1452

76 Marrus N, Eggebrecht A T, Todorov A, et al. Walking, gross motor development, and brain functional connectivity in infants and toddlers. Cereb Cortex, 2018, 28: 750-763

77 Geng D, Zhang X, Shi J. The development of fine motor skills and its relation to cognitive development in young children. Adv Psychol Sci, 2015, 23: 261-267

78 Bayer U, Hausmann M. Menstrual cycle-related changes of functional cerebral asymmetries in fine motor coordination. Brain Cogn, 2012, 79: 34-38

79 Gooijers J, Leemans A, Van Cauter S, et al. White matter organization in relation to upper limb motor control in healthy subjects: exploring the added value of diffusion kurtosis imaging. Brain Struct Funct, 2014, 219: 1627-1638

80 Roberts R E, Bain P G, Day B L, et al. Individual differences in expert motor coordination associated with white matter microstructure in the cerebellum. Cereb Cortex, 2013, 23: 2282-2292

81 Grohs M N, Reynolds J E, Dewey D, et al. Corpus callosum microstructure is associated with motor function in preschool children. NeuroImage, 2018, 183: 828-835

82 Jäncke L, Koeneke S, Hoppe A, et al. The architecture of the golfer's brain. PLoS ONE, 2009, 4: e4785

83 Fatemi S H, Aldinger K A, Ashwood P, et al. Consensus paper: pathological role of the cerebellum in autism. Cerebellum, 2012, 11: 777-807

84 Mostofsky S H, Rimrodt S L, Schafer J G B, et al. Atypical motor and sensory cortex activation in attention-deficit/hyperactivity disorder: A functional magnetic resonance imaging study of simple sequential finger tapping. Biol Psychiatry, 2006, 59: 48-56

85 Gabbard C, Caçola P, Bobbio T. Examining age-related movement representations for sequential (fine-motor) finger movements. Brain Cogn, 2011, 77: 459-463

86 Li F, Yan C H, Shen X M. Research progress of early fine motor skill development promoting brain cognitive development (in Chinese). Natl Med J China, 2005, 85: 2157-2159 [李斐, 颜崇淮, 沈晓明. 早期精细动作技能发育促进脑认知发展的研究进展. 中华医学杂志, 2005, 85: 2157-2159]

87 Cameron C E, Brock L L, Murrah W M, et al. Fine motor skills and executive function both contribute to kindergarten achievement. Child Dev, 2012, 83: 1229-1244

88 Batouli S A H, Saba V. At least eighty percent of brain grey matter is modifiable by physical activity: A review study. Behav Brain Res, 2017, 332: 204-217

89 Esteban-Cornejo I, Catena A, Hillman C H, et al. Commentary: At least eighty percent of brain grey matter is modifiable by physical activity: a review study. Front Hum Neurosci, 2018, 12: 195

90 Seidler R, Erdeniz B, Koppelmans V, et al. Associations between age, motor function, and resting state sensorimotor network connectivity in healthy older adults. NeuroImage, 2015, 108: 47-59

91 Zielinski B A, Gennatas E D, Zhou J, et al. Network-level structural covariance in the developing brain. Proc Natl Acad Sci USA, 2010, 107: 18191-18196

92 Ridler K, Veijola J M, Tanskanen P, et al. Fronto-cerebellar systems are associated with infant motor and adult executive functions in healthy adults but not in schizophrenia. Proc Natl Acad Sci USA, 2006, 103: 15651-15656

93 Rigoli D, Piek J P, Kane R, et al. An examination of the relationship between motor coordination and executive functions in adolescents. Dev Med Child Neurol, 2012, 54: 1025-1031

94 Tandon P S, Tovar A, Jayasuriya A T, et al. The relationship between physical activity and diet and young children's cognitive development: A systematic review. Prev Med Rep, 2016, 3: 379-390

95 Carson V, Hunter S, Kuzik N, et al. Systematic review of physical activity and cognitive development in early childhood. J Sci Med Sport, 2016, 19: $573-578$

96 Jackson W M, Davis N, Sands S A, et al. Physical activity and cognitive development: a meta-analysis. J Neurosurg Anesthesiol, 2016, 28: 373380

97 Zeng N, Ayyub M, Sun H, et al. Effects of physical activity on motor skills and cognitive development in early childhood: a systematic review. Biomed Res Int, 2017, 2017: 1-13

98 Li L, Zhang J, Cao M, et al. The effects of chronic physical activity interventions on executive functions in children aged 3-7 years: A metaanalysis. J Sci Med Sport, 2020, 23: 949-954 
99 Sexton C E, Betts J F, Demnitz N, et al. A systematic review of MRI studies examining the relationship between physical fitness and activity and the white matter of the ageing brain. NeuroImage, 2016, 131: 81-90

100 Valkenborghs S R, Noetel M, Hillman C H, et al. The impact of physical activity on brain structure and function in youth: a systematic review. Pediatrics, 2019, 144: e20184032

101 Pangelinan M M, Zhang G, VanMeter J W, et al. Beyond age and gender: Relationships between cortical and subcortical brain volume and cognitive-motor abilities in school-age children. NeuroImage, 2011, 54: 3093-3100

102 Seidel O, Ragert P. Effects of transcranial direct current stimulation of primary motor cortex on reaction time and tapping performance: a comparison between athletes and non-athletes. Front Hum Neurosci, 2019, 13

103 Ren Z B, Hu L L, Zhang Y C, et al. A review of brain plasticity of motor skill experts: evidence from magnetic resonance imaging (in Chinese). China Sport Sci Technol, 2019, 55: 3-18 [任占兵, 胡琳琳, 张远超, 等. 运动技能专家脑可塑性研究进展: 来自磁共振成像的证据. 中国体 育科技, 2019, 55: 3-18]

104 Shao M, Lin H, Yin D, et al. Learning to play badminton altered resting-state activity and functional connectivity of the cerebellar sub-regions in adults. PLoS ONE, 2019, 14: e0223234

105 Park I S, Lee K J, Han J W, et al. Experience-dependent plasticity of cerebellar vermis in basketball players. Cerebellum, 2009, 8: 334-339

106 Tao Z P, Gao Q, Yu Y, et al. A study of brain plasticity of small-ball players based on ReHo method (in Chinese). J Chengdu Sport, 2017, 43: 98-102 [陶中平, 高晴, 余意, 等. 基于局部一致性方法的小球类运动员脑可塑性变化研究. 成都体育学院学报, 2017, 43: 98-102]

107 Bai X J, Shao M L, Liu T, et al. Altered structural plasticity in early adulthood after badminton training (in Chinese). Acta Psychol Sin, 2020, 52: 173-183 [白学军, 邵梦灵, 刘婷, 等. 羽毛球运动重塑成年早期的大脑灰质和白质结构. 心理学报, 2020, 52: 173-183]

108 Zhang Y, Zhao L, Bi W, et al. Effects of long-term diving training on cortical gyrification. Sci Rep, 2016, 6: 28243

109 Wei G, Luo J, Li Y. Brain structure in diving players on MR imaging studied with voxel-based morphometry. Prog Nat Sci, 2009, 19: 13971402

110 Tremblay S, Pascual-Leone A, Théoret H. A review of the effects of physical activity and sports concussion on brain function and anatomy. Int J Psychophysiol, 2018, 132: 167-175

111 Freund W, Faust S, Gaser C, et al. Regionally accentuated reversible brain grey matter reduction in ultra marathon runners detected by voxelbased morphometry. BMC Sports Sci Med Rehabil, 2014, 6: 4

112 Duru A D, Balcioglu T H. Functional and structural plasticity of brain in elite karate athletes. J Healthc Eng, 2018, 2018: 1-8

113 Boorman E D, O'Shea J, Sebastian C, et al. Individual differences in white-matter microstructure reflect variation in functional connectivity during choice. Curr Biol, 2007, 17: 1426-1431

114 O'Shea J, Sebastian C, Boorman E D, et al. Functional specificity of human premotor-motor cortical interactions during action selection. Eur J Neurosci, 2007, 26: 2085-2095

115 Wadden K, Brown K, Maletsky R, et al. Correlations between brain activity and components of motor learning in middle-aged adults: an fMRI study. Front Hum Neurosci, 2013, 7: 169-179

116 Hoffstaedter F, Grefkes C, Roski C, et al. Age-related decrease of functional connectivity additional to gray matter atrophy in a network for movement initiation. Brain Struct Funct, 2015, 220: 999-1012

117 Hirsiger S, Koppelmans V, Mérillat S, et al. Structural and functional connectivity in healthy aging: Associations for cognition and motor behavior. Hum Brain Mapp, 2016, 37: 855-867

118 Tomasi D, Volkow N D. Aging and functional brain networks. Mol Psychiatry, 2012, 17: 549-558

119 He H, Luo C, Chang X, et al. The functional integration in the sensory-motor system predicts aging in healthy older adults. Front Aging Neurosci, 2017, 8

120 Lee E Y, Sen S, Eslinger P J, et al. Early cortical gray matter loss and cognitive correlates in non-demented Parkinson's patients. Parkinsonism Relat Disord, 2013, 19: 1088-1093

121 Wood K N, Nikolov R, Shoemaker J K. Impact of long-term endurance training vs. guideline-Based physical activity on brain structure in healthy aging. Front Aging Neurosci, 2016, 8

122 Chang M, Jonsson P V, Snaedal J, et al. The effect of midlife physical activity on cognitive function among older adults: AGES-Reykjavik study. J Gerontol A Biol Sci Med Sci, 2010, 65A: 1369-1374

123 Voss M W, Heo S, Prakash R S, et al. The influence of aerobic fitness on cerebral white matter integrity and cognitive function in older adults: 
results of a one-year exercise intervention. Hum Brain Mapp, 2013, 34: 2972-2985

124 Marks B L, Katz L M, Styner M, et al. Aerobic fitness and obesity: relationship to cerebral white matter integrity in the brain of active and sedentary older adults. Br J Sports Med, 2011, 45: 1208-1215

125 Firth J, Stubbs B, Vancampfort D, et al. Effect of aerobic exercise on hippocampal volume in humans: A systematic review and meta-analysis. NeuroImage, 2018, 166: 230-238

126 Wei G X, Song X F, Huang T, et al. Trends of MRI-based sport experts brain plasticity: A bibliometric study (in Chinese). J Wuhan Inst Phys Ed, 2020, 54: 63-70 [魏高峡, 宋秀芳, 黄涛, 等. 基于磁共振成像技术的运动员大脑可塑性研究: 发展态势与热点分析. 武汉体育学院学报, 2020, 54: 63-70]

\title{
The neuroscience of motor development: the future path and layout
}

\author{
WU ZhiJun ${ }^{1,2}$, WANG ZhengYan ${ }^{1,2} \&$ WANG Qiang ${ }^{1,2}$ \\ 1 Beijing Key Laboratory of Learning and Cognition, School of Psychology, Capital Normal University, Beijing 100048, China; \\ 2 Child Development Research Center, School of Psychology, Capital Normal University, Beijing 100048, China
}

The development of children's early motor can be regarded as an individual's early explicit intelligence, and the lifelong development of motor is closely related to an individual's cognitive development, and personality and social development. The revolutionary development of cognitive neuroscience and brain science technology provides the foundation and opportunity for studying neural mechanisms of lifelong development in the field of motor. From the perspective of lifelong development, the generation of motor and the acquisition of motor skills are milestones of infant development. The effectiveness, accuracy and stability of motor skills in childhood and adolescence develop rapidly. From the early to late adulthood, motor ability experiences a peak and then a significant decline. Current research on neural mechanisms of actions mainly discusses the changes in brain structure and brain network connections behind the generation, development and decline of actions during the lifelong development. From the perspective of cognitive science and cognitive neuroscience, this article reviewed the developmental characteristics of individual motor in infancy, child, adolescence and adulthood and neural mechanisms behind it. It also put forward the challenges and problems to be solved in the future of cognitive neuroscience, and analyzed and predicted the future research and development in this field in China.

motor, lifelong development, cognitive psychology, cognitive neuroscience

doi: $10.1360 / \mathrm{SSV}-2020-0242$ 